\title{
On the Multicomponent Polynomial Solution Models
}

\author{
Dmitry Saulov ${ }^{\mathrm{a}}$
}

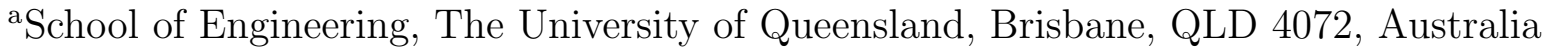
Tel.: (61-7)3365-6073; Fax: (61-7)3365-3670; E-mail address: d.saulov@uq.edu.au

\begin{abstract}
The present study addresses the problem of predicting the properties of multicomponent systems from those of corresponding binary systems. Two types of multicomponent Polynomial models have been analysed. A probabilistic interpretation of the parameters of the Polynomial model, which explicitly relates them with the Gibbs free energies of the generalised quasichemical reactions, is proposed. The presented treatment provides a theoretical justification for such parameters. A methodology of estimating the ternary interaction parameter from the binary ones is presented. The methodology provides a way, in which the power series multicomponent models, where no projection is required, could be incorporated into the Calphad approach.
\end{abstract}

Keywords: Power series models; Projected models; Calphad approach; Probabilistic interpretation; Generalised quasichemical reaction.

\section{Introduction}

A polynomial representation of the excess Gibbs free energy (or the Polynomial solution model) dated from, at least, the work by Max Margules [1] published in 1895. The coefficients of the polynomial expansion are often called Margules' parameters. Despite the availability of more sophisticated solution models, the Polynomial model still widely used to describe the thermodynamic properties of solutions of metallurgical, geological, 
and chemical engineering interest. Many binary systems can be described reasonably well with the polynomial solution model. However, the long-standing problem of predicting the properties of multicomponent systems from those of corresponding binaries remains. Cheng and Ganguly [2], for example, regarded this problem as "a major problem in solution thermodynamics".

Many methods (or models) have been suggested to address the problem. According to Cheng and Ganguly [2], all these models can be divided into two groups: power series multicomponent models that are based on the Taylor series expansion of the multicomponent excess Gibbs free energy surface, and projected multicomponent models, in which interaction parameters in a multicomponent system are determined by projections of the multicomponent composition to corresponding binary lines. The latter group is also referred to as geometric [3, 4] or numeric [5] models.

One of the earliest and most successful studies on the multicomponent models related to the first group is the work by Wohl [6]. Wohl extensively discussed the models available at that time and presented them in a unified way as "variations of one mathematical scheme". Helffrich and Wood [7] and later Mukhopadhyay et al. [8] and Cheng and Ganguly [2] generalised the Wohl's approach for arbitrary number of the solution components. Mukhopadhyay et al. [8] extended the approach to arbitrary degree of polynomials, reviewed the recent formulations of multicomponent models of the first group (see the work by Mukhopadhyay et al. [8] and references therein), and established their equivalence with the Wohl's formulation.

Different models of the second group have also been suggested. However, only few of them, namely Kohler, Muggianu, Kohler/Toop, and Muggianu/Toop models are widely used. The fist two of these models are symmetric, while the others are asymmetric. These models have been extensively reviewed in the literature (see, for example, $[5,9]$ ). Less popular models, as well as recently developed models (see the work by Ganesan and Varamban [10] and references therein) are not considered in the present study. 
The projected multicomponent models are an essential part of the approach used by international Calphad group, where, as pointed out by Pelton [11], "interpolation procedures based on solution models are used to estimate the Gibbs free energies of the ternary phases from the Gibbs free energies of the binary phases". In contrast, the power series multicomponent models do not seem to be directly in line with the Calphad approach, since the information about the multicomponent excess Gibbs free energy surface is required to obtain the coefficients of the Taylor series expansion. However, a way in which the power series models could be incorporated in the Calphad approach is suggested in the present study.

\section{Terminology Used}

In the present study, the interactions that occur in a binary system are referred to as binary interactions. The binary interactions should be clearly distinguished from twobody interactions which are those between two mixing particles (atoms, ions, molecules, etc.) in a solution. As described, for example, by Hillert [9], the pair-exchange reaction $\left(A_{1}-A_{1}\right)+\left(A_{2}-A_{2}\right)=2\left(A_{1}-A_{2}\right)$ between two-body interactions (interpreted as bonds) of different types is considered in the Regular solution model. The molar excess Gibbs free energy is expressed as follows:

$$
{ }^{E} g\left(x_{1}, x_{2}\right)=\alpha_{12} x_{1} x_{2}=N^{0} z \Delta g_{12} x_{1} x_{2} / 2
$$

Here, $N^{0}$ is the Avogadro number, $z$ is the coordination number, which is the average number of nearest-neighbours per particle. $\Delta g_{12}$ is the Gibbs free energy change in forming two moles of $\left(A_{1}-A_{2}\right)$ nearest-neighbour pairs from one mole of $\left(A_{1}-A_{1}\right)$ pairs and one mole of $\left(A_{2}-A_{2}\right)$ pairs, and $x_{1}$ and $x_{2}$ are the mole fractions of the components $A_{1}$ and $A_{2}$ respectively.

The binary interaction parameter $\alpha_{12}$, which is the coefficient before the product $x_{1} x_{2}$, here is $N^{0} z \Delta g_{12} / 2$. In the case of the Regular model, where only two-body interactions 
are considered, the binary interaction parameter does not depend on composition. On the other hand, in the Subregular solution model the binary interaction parameter does depend on composition. According to Wohl [6], this compositional dependence can be related to the three-body interactions such as $\left(A_{1}-A_{1}-A_{2}\right)$ and $\left(A_{1}-A_{2}-A_{2}\right)$ in the binary system $A_{1}-A_{2}$. The coefficient $\alpha_{123}$ in the ternary term $\alpha_{123} x_{1} x_{2} x_{3}$ is referred to as the ternary interaction parameter in the present study.

\section{Shortcomings of the Projected Multicomponent Models}

Many authors, for example [2, 5, 12], expressed a preference in favour of the Muggianu model. However, Chartrand and Pelton [3] disagreed "with the current tendency to use the symmetric Muggianu model for nearly all solutions". They extensively discussed the advantages and disadvantages of the different geometric models and concluded that the Kohler model is more preferable than the Muggianu model and the asymmetric models are more appropriate for the systems in which one of the components behaves chemically different from the other two. Chartrand and Pelton also proposed the equations for extending the symmetric/asymmetric dichotomy into multicomponent systems. The arguments presented by Chartrand and Pelton [3], as well as the reasoning of other authors are discussed below.

\subsection{Kohler vs Muggianu}

Chartrand and Pelton [3] used the example of a dilute solution to justify their preference in favour of the Kohler model. Following Chartrand and Pelton [3], if a ternary solution $A_{1}-A_{2}-A_{3}$ of composition $p$ is dilute in the component $A_{1}$, as illustrated in Fig. 1 , the Kohler model uses the values for the binary interaction parameters $\alpha_{12}$ and $\alpha_{13}$ at the compositions $a^{\prime}$ and $b^{\prime}$ in the binary systems $A_{1}-A_{2}$ and $A_{1}-A_{3}$ respectively which are also dilute in the component $A_{1}$. The Muggianu model utilises the values of the binary interaction parameters at the compositions $a$ and $b$ which are not dilute in the component $A_{1}$. No other arguments in support of Kohler model have been found in the work by 


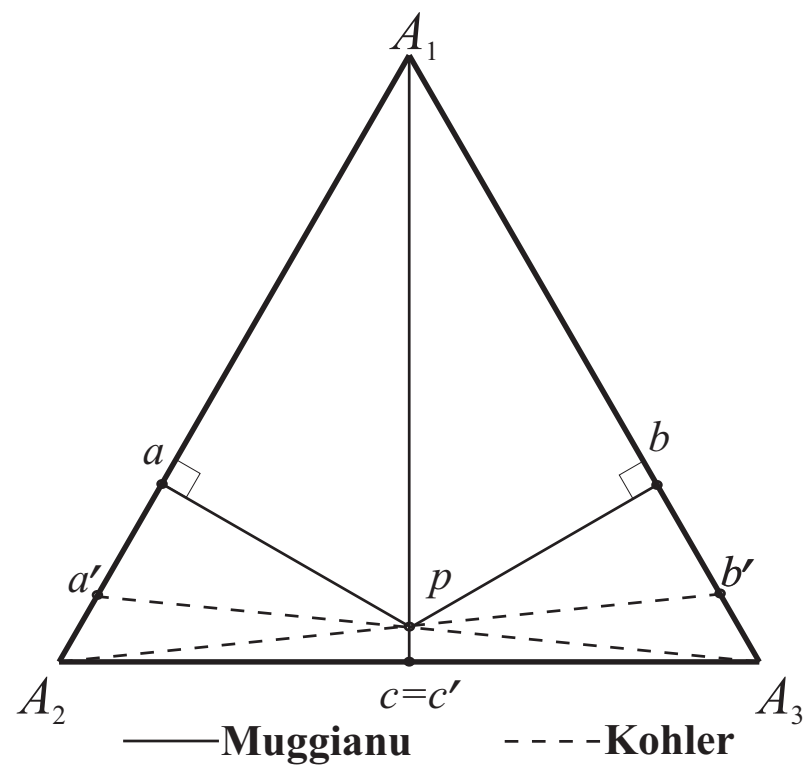

Figure 1. The Kohler model vs the Muggianu model applied to a ternary solution $A_{1}-$ $A_{2}-A_{3}$ that dilute in the component $A_{1}$.

Chartrand and Pelton [3].

The dilute solution consideration is of practical importance, for example, when only those parts of the systems $A_{1}-A_{2}$ and $A_{1}-A_{3}$ which are dilute in the component $A_{1}$, are experimentally investigated. In this case, the expressions used for the binary interaction parameters could be unreliable for the compositions concentrated in $A_{1}$. At the same time, it is desirable to use the values from the reliable composition regions. However, as shown in Fig. 2, if the ternary composition $p$ dilute in both $A_{1}$ and $A_{2}$, the Kohler model employs even less reliable value of the binary interaction parameter in the system $A_{1}-A_{2}$.

The dilute solution consideration applied to the Kohler/Toop model, which has also been recommended by Chartrand and Pelton [3], is illustrated in Fig. 3. As shown in Fig. 3 , for the ternary solution that is dilute in the component $A_{2}$ the Kohler/Toop model utilises the value of the binary interaction parameter at the composition $a$ that is even more concentrated in $A_{2}$ than the composition $a^{\prime}$ used in the Muggianu model. 


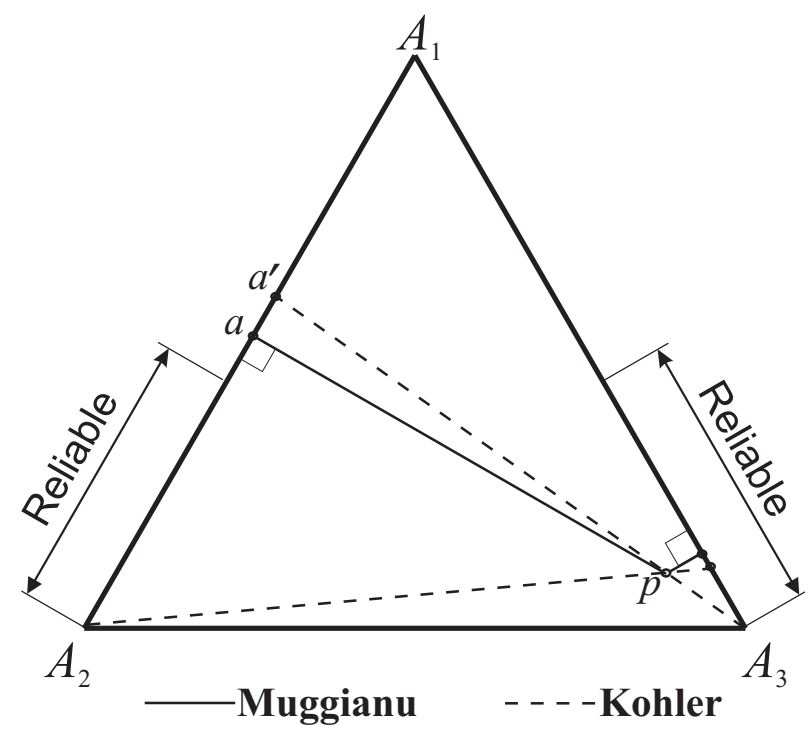

Figure 2. The Kohler model vs the Muggianu model applied for the solution that dilute in both the component $A_{1}$ and the component $A_{2}$.

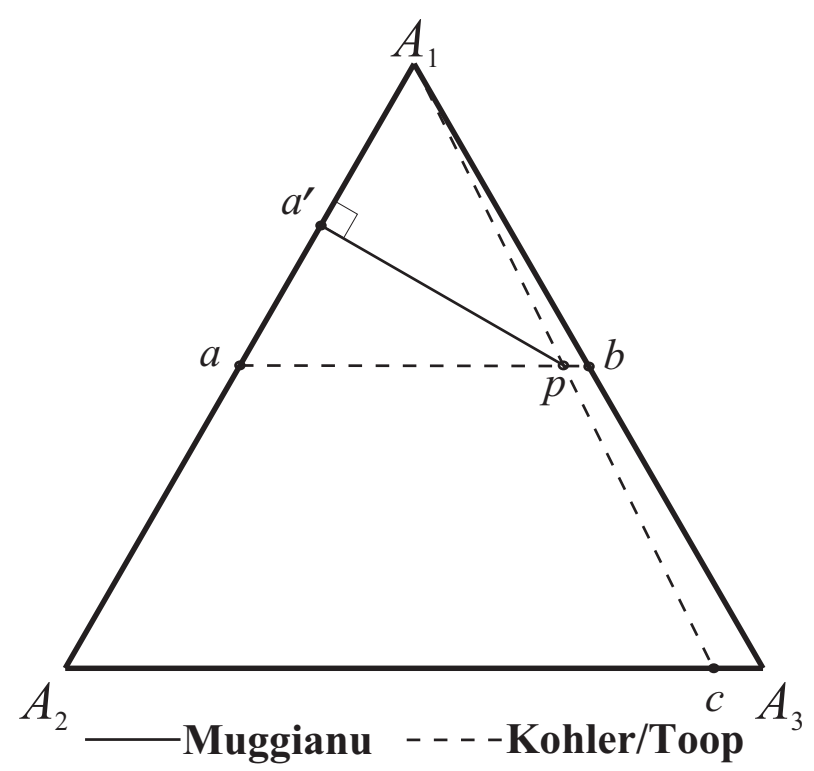

Figure 3. The Kohler/Toop model applied for the ternary solution that dilute in the component $A_{2}$. 
Brynestad [13] demonstrated that the Kohler model combined with the Subregular solution model leads to discontinuities in the derivatives of the partial excess Gibbs free energy at terminal compositions. Brynestad [13] concluded that the Kohler model is incompatible with the Subregular solution model. Kaufman [14] agreed with the Brynestad's mathematics but disagreed with his conclusion. In agreement with Kaufman, Hillert and Sundman [15] stated that the "existence of this singular point is of no physical consequence because it only concerns the property of a component in a point where that component is absent".

However, assume that the Kohler model is used for modelling a ternary liquid solution $A_{1}-A_{2}-A_{3}$, and define the function $F\left(T, x_{1}, x_{2}, x_{3}\right)$ as follows:

$$
F\left(T, x_{1}, x_{2}, x_{3}\right) \equiv g_{1}^{0 ; \text { sol }}(T)-\mu_{1}^{\operatorname{liq}}\left(T, x_{1}, x_{2}, x_{3}\right)
$$

Here, $g_{1}^{0 ; \text { sol }}$ is the molar Gibbs free energy of pure solid $A_{1}$, and $\mu_{1}^{\text {liq }}$ is the chemical potential of the component $A_{1}$ in the liquid of composition $\left(x_{1}, x_{2}, x_{3}\right)$. Using the well-known expression for the chemical potential (see, for example [9]), the function $F$ defined by Eq. (2) can be expressed in terms of partial Gibbs free energies. The equation $F\left(T, x_{1}, \ldots, x_{k}\right)=0$ gives an implicit functional relation between the liquidus temperature $T$ and the composition of the liquid $\left(x_{1}, x_{2}, x_{3}\right)$ in the primary phase field of the component $A_{1}$. If all the conditions of the implicit function theorem are satisfied, the gradient of the liquidus can be calculated from the following equations:

$$
\frac{\partial T}{\partial x_{i}}=-\frac{\partial F\left(T, x_{1}, x_{2}, x_{3}\right) / \partial x_{i}}{\partial F\left(T, x_{1}, x_{2}, x_{3}\right) / \partial T} \quad(i=1, \ldots, 3)
$$

Eqs. 3 include the derivatives of the partial excess Gibbs free energies which, as shown by Brynestad [13], have singularities at terminal compositions. In other words, the Kohler model applied to subregular binaries could result in non-smooth predictions of the liquidus surface which is a matter of concern. Howald and Row [16] also discussed different 
extrapolation methods and came to the conclusion that the Kohler model should not be used. As pointed out by Howald and Row, many extrapolation models are available which do not have the inconsistency of the Kohler model and will fit the experimental data with the same or better quality.

The Muggianu model uses the values of the binary interaction parameters that are evaluated at the points on the corresponding binaries which are geometrically closest to the ternary composition in the sense of standard Euclidean distance. Despite the expressed preference in favour of the Kohler model, Chartrand and Pelton [3] pointed out that the approach used in the Muggianu model seems intuitively more justifiable from a physical standpoint, than one used in the Kohler model, where the binary interaction parameter is assumed to be constant at constant $x_{i} /\left(x_{i}+x_{j}\right)$ ratio. Cheng and Ganguly [2] pointed out that the Muggianu model constitutes the most rational approach, since it also follows from the second or third degree Taylor series expansion of the multicomponent Gibbs free energy surface. In contrast, the Kohler model can not be reconciled with the Taylor series expansion of the multicomponent Gibbs free energy surface.

\subsection{Symmetric vs Asymmetric Projected Models}

Chartrand and Pelton [3] stated that if one of the components in a ternary system is chemically different while the other two are chemically similar, then an asymmetric model is more physically reasonable than a symmetric one. To support this statement Chartrand and Pelton provided an example of a ternary system $A_{1}-A_{2}-A_{3}$ in which the binary $A_{2}-A_{3}$ is ideal, while the binaries $A_{1}-A_{2}$ and $A_{1}-A_{3}$ exhibit subregular behaviour with the identical binary interaction parameters $\alpha_{12}=\alpha_{13}=-50\left(1-x_{1}\right) \mathrm{kJ} / \mathrm{mol}$. If the binary systems exhibit the described behaviour, the molar excess Gibbs free energy of the ternary phase is expected to be constant at constant $x_{1}$. However, the symmetric Kohler model fails to predict this behaviour. Instead, it predicts a spurious miscibility gap (see Fig. 3 in [3]). According to Chartrand and Pelton [3], the symmetric Muggianu model gives similarly incorrect predictions, while the asymmetric Kohler/Toop method resolves 
this particular discrepancy.

However, the development of quantitative criteria for selecting an asymmetric component remains an important open problem. Indeed, without such criteria a modeller should arbitrarily decide which component (if any) is to be treated asymmetrically, when the components do not differ dramatically. Such a decision could be made based on the available ternary experimental data. Nevertheless, there is no guarantee that the decision made will provide a satisfactory fit for another ternary dataset. Therefore, the lack of the quantitative criteria leaves some subjectivity in the aforementioned decision.

Furthermore, the possibility to treat different phenomena within the unified formalism constitutes one of the major advances of Thermodynamics. From this standpoint, it would be desirable to treat all the components in a thermodynamically unified way, even if they are dissimilar chemically. As demonstrated by Hillert [5] and later by Cheng and Ganguly [2], the problem of spurious miscibility gap is resolved in the symmetric Muggianu model with the help of appropriately selected ternary interaction parameter. Note that all the solution components are treated in a unified way in the symmetric Muggianu model. Another alternative method to solve the problem in a thermodynamically unified way is presented in Section 6.1 of the present study.

\section{Projected Models vs Power Series Models}

As discussed above, if only two-body interactions are considered, the binary interaction parameter does not depend on composition. As soon as the variation of the binary interaction parameter with composition is allowed, as it is in the Subregular solution model, it is implicitly assumed that the system could not be described in terms of twobody interactions only, and three-body interactions are involved. However, in this case, the three-body interaction between particles of all types in the ternary system should also be considered. These three-body and three-component interactions accounted for by the ternary term. 
The variation of the binary interaction parameter with composition could be attributed to the influence of the "environment" on the two-body interactions. Again, the multi-body interactions are implicitly assumed. In projected multicomponent models the influence of the binary environment on the two-body interactions is projected into the ternary system. However, the environment in the ternary system principally differs from the environment in any of the bounding binary systems, and the ternary environment can not be predicted from the binary environment only. In other words, an appropriate ternary term should be incorporated to account for the specific of the ternary environment in predicting the behaviour of the ternary system.

Helffrich and Wood [7] demonstrated that for subregular solutions ternary term exist independently of the properties of the bounding binaries. This can be considered as an indication that the ternary terms should always be considered for subregular binaries. Moreover, it is also demonstrated by Helffrich and Wood [7] that the ternary term could be required even if the bounding binaries are describable with the Regular solution model. The problem is that the regular behaviour of the binary system can not be distinguished from the special symmetric case of the subregular behaviour based on the measurements in the binary system only. Indeed, let us assume that the binary system $A_{1}-A_{2}$ behaves regularly. In this case, the molar excess Gibbs free energy is expressed by Eq. (1). Since $x_{1}+x_{2} \equiv 1$ in the binary system, Eq. (1) can be rearranged as follows: ${ }^{E} g\left(x_{1}, x_{2}\right)=$ $\alpha_{12} x_{1} x_{2}\left(x_{1}+x_{2}\right)=\alpha_{12} x_{1}^{2} x_{2}+\alpha_{12} x_{1} x_{2}^{2}$. The same considerations are valid for the binaries $A_{1}-A_{3}$ and $A_{2}-A_{3}$. If the binary systems exhibit the symmetric case of subregular behaviour, the appropriate ternary term is required in the ternary system. However, to discriminate between the regular and the symmetric subregular behaviour, independent measurements in the ternary system are necessary. Similar treatment is valid for an arbitrary number of the solution components and an arbitrary degree of polynomials.

The ternary and multicomponent terms that are required for reasonable prediction of the behaviour of ternary and multicomponent systems are an integral part of the power 
series models. In the projected models, such terms are considered as empirical terms that could be used to fit ternary experimental data, where such data are available. Chartrand and Pelton [3], for example, affirmed that the proper projected model should provide a reasonable first estimate of the ternary excess Gibbs free energy without any ternary terms which "have little theoretical justification". Indeed, if only two-body interactions are considered, ternary terms are irrelevant. In this case, however, the binary interaction parameters are independent of the composition, and no projection is required. Nevertheless, Chartrand and Pelton agreed that the ternary terms "might be related to the energies of triplet interactions". They also designed a special form for these terms to represent the effect of a third component upon the energy of the pair exchange reaction.

The probabilistic interpretation of the coefficients of the power series multicomponent model presented in the next section explicitly relates the ternary and multicomponent interaction parameters with three-body and multi-body interactions. The presented treatment provides a theoretical justification for such parameters. This interpretation can be considered as a generalisation of the Regular solution model, where only two-body interactions are considered, for multi-body interactions in binary and multicomponent systems.

\section{Probabilistic Interpretation of the Parameters of the Power Series Multi- component Model}

As pointed out by Hillert [9], a simple but useful way of modelling the thermodynamic properties of solutions is based on the assumption that the energy of the whole system is the sum of the energies of interactions between neighbouring particles (atoms, molecules, ions). In the simplest case of the Regular solution model, the energies of nearest neighbour pairs are only considered, and random mixing of the particles over the equivalent sites of a lattice is assumed. The formation of a solution can then be regarded as the quasichemical reaction between pairs of different kinds. 
However, many real solutions exhibit a more complex behaviour which indicates that the energies of triplets, quadruplets, or even more complex agglomerates of mixing particles should be considered. Such solutions can be described reasonably well by the Polynomial solution model. A general treatment that relates the Polynomial model parameters with the energies of generalised quasichemical reactions is presented below. The assumptions underlying this treatment are formulated in the next section.

\subsection{Assumptions Used}

\section{Random mixing}

The most important assumption which is extensively used in the present treatment is that of random mixing of particles over the lattice sites. This assumption is also known as the Bragg-Williams approximation. More precisely, the random mixing assumption consists in the following:

- the occupancy of any site is stochastically independent from the occupancies of all the other sites;

- the probability distribution of the occupancy of a site (by particles of different types) is the same for all lattice sites.

\section{Short range interactions under consideration}

Similarly to the derivation of the Regular solution model presented by Hillert [9], only the energies of neighbouring particles are considered, with the neighbouhood defined immediately below. This assumption is related to the fact that the interaction energy decays with increasing the distance between interacting particles. When only two-body interactions are considered, a threshold distance can be defined. If the distance between two sites of the lattice exceeds the threshold, the energy of interaction between particles at these sites is neglected.

However, it is not clear how the threshold should be defined for multi-body interactions. Different approaches are possible. The approach used in the present study is elucidated 


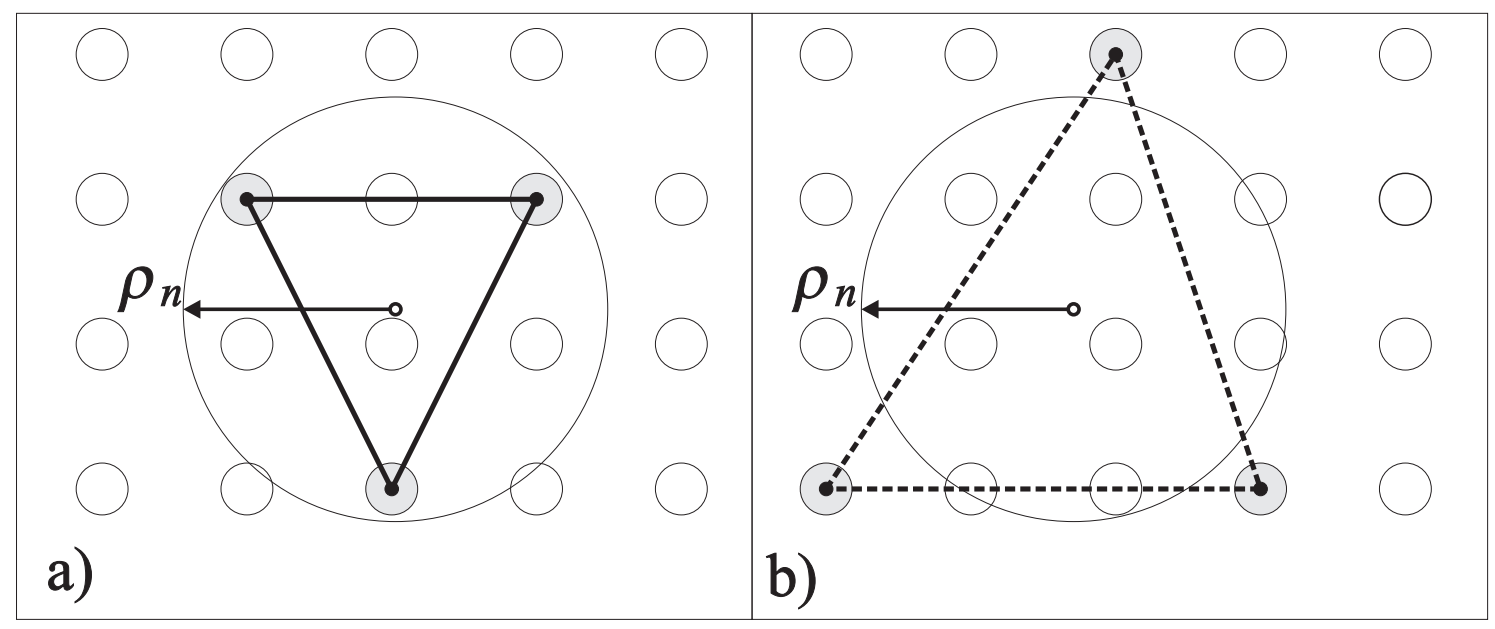

Figure 4. a) Interacting sites. The sites can be covered with the sphere of interaction of the threshold radius $\rho_{n}$. b) Non-interacting sites.

in Fig. 4. A set of $n$ different lattice sites is called an $n$-clique if this set is contained entirely in a sphere of radius $\rho_{n}$ as illustrated in Fig. 4 a). Such sphere will be referred to as an interaction sphere, and the particles occupying the sites of an $n$-clique will be interpreted as an $n$-agglomerate of neighbouring particles which interact with each other. If a set of lattice sites is not contained by an interaction sphere of the threshold radius $\rho_{n}$, as schematically presented in Fig. $4 \mathrm{~b}$ ), the interaction energy between particles at these sites is neglected. The threshold radius $\rho_{n}$ is not used explicitly in the presented treatment, although its existence is assumed.

Clearly, the interaction sphere should be large enough to enclose $n$ or more sites. On the other hand, the interacting sites are not necessarily the nearest neighbours for the relatively large threshold radius. Note also that the centre of the interaction sphere not necessarily coincides with a lattice site.

\section{Properties of the lattice}

The third group of the assumptions made in the present study is related to the properties of the lattice. It is assumed that all the lattice sites are equivalent, and the spatial arrangement of the sites is not influenced by their occupancies. Under these assumptions 
the average number $z_{n}$ of $n$-cliques, in which a particular site participates, is the same for each site and independent from the occupancies of the sites. $z_{n}$ depends on the threshold radius $\rho_{n}$ of the sphere of interaction. However, this dependence does not used in the presented treatment explicitly.

The assumptions on the lattice made do not hold for complex crystalline solutions, in which particles are mixed over several different sub-lattices, as well as for those liquid solutions, in which particles of different solution components significantly differ in size. However, the Polynomial model could provide preliminary approximation even in these more complex cases.

\subsection{Derivation}

Consider an $r$-component solution $A_{1}-A_{2}-\ldots-A_{r}$ and assume that the Gibbs free energy of the solution can be described as the sum of the Gibbs free energies of $n$-agglomerates of particles and the ideal entropy term. The Gibbs free energy of an $n$ agglomerate depends on the types of constituent particles and their spatial arrangement within the $n$-clique of sites.

Now consider an $n$-agglomerate that consists of $k_{1}$ particles of type $A_{1}, k_{2}$ particles of type $A_{2}$ and so on, where $k_{1}+\ldots+k_{r}=n$, so that the numbers $k_{1}, \ldots, k_{r}$ specify the "chemical composition" of the agglomerate. The particles in the agglomerate can have different spatial arrangements with different values of Gibbs free energy. Denote by $g_{n ; k_{1}, \ldots, k_{r}}$ the conditional average of non-configurational Gibbs free energy of the $n$-agglomerate, where the average is taken over all possible spatial arrangements, provided that the "chemical composition" of the agglomerate is $k_{1}, \ldots, k_{r}$. The entropy part of $g_{n ; k_{1}, \ldots, k_{r}}$ takes into account only non-configurational (for example, vibrational and rotational) degrees of freedom.

In the framework of the Bragg-Williams approximation, the "chemical composition" of a randomly selected $n$-agglomerate follows the multinomial distribution [17] with the parameters $n, x_{1}, \ldots, x_{r}$, where $x_{1}, \ldots, x_{r}$ are the molar fractions of the solution compo- 
nents $A_{1}, \ldots, A_{r}$ respectively. More precisely, the probability $p_{n ; k_{1}, \ldots, k_{r}}$ to pick up an $n$ agglomerate of a given "chemical composition" $k_{1}, \ldots, k_{r}$ is given by

$$
p_{n ; k_{1}, \ldots, k_{r}}=\frac{n !}{k_{1} ! \ldots k_{r} !} x_{1}^{k_{1}} \ldots x_{r}^{k_{r}}
$$

Then the average non-configurational Gibbs free energy $\left\langle g_{n}\right\rangle$ of a randomly chosen $n$ agglomerate is expressed as

$$
\left\langle g_{n}\right\rangle=\sum_{k_{1}+\ldots+k_{r}=n} \frac{n !}{k_{1} ! \ldots k_{r} !} x_{1}^{k_{1}} \ldots x_{r}^{k_{r}} g_{n ; k_{1}, \ldots, k_{r}}
$$

where the sum is taken over all ordered collections of nonnegative integers $k_{1}, \ldots, k_{r}$ such that $k_{1}+\ldots+k_{r}=n$.

Under the assumptions made on the lattice properties, the average number $z_{n}$ of $n$ cliques containing a given site is independent of the spatial position of the site. The average number of all $n$-cliques in one mole of a solution $\left(N^{0}\right.$ particles $)$ is $z_{n} N^{0} / n$. Here, the factor $1 / n$ appears since each $n$-clique is counted precisely $n$ times in the product $z_{n} N^{0}$. Then, the non-configurational molar Gibbs free energy $g^{\text {nc }}$ of the solution is expressed as

$$
g^{\mathrm{nc}}=\frac{z_{n} N^{0}}{n}\left\langle g_{n}\right\rangle=\frac{z_{n} N^{0}}{n}\left(\sum_{k_{1}+\ldots+k_{r}=n} \frac{n !}{k_{1} ! \ldots k_{r} !} x_{1}^{k_{1}} \ldots x_{r}^{k_{r}} g_{n ; k_{1}, \ldots, k_{r}}\right)
$$

All energetic interactions between neighbouring particles are counted in Eq. (6). Consider, for example, two $n$-agglomerates $\mathrm{A}$ and $\mathrm{B}$. The agglomerates could be spatially close or even share several particles and, therefore, interact with each other. However, the energy of interaction between the agglomerate A and B is accounted for in a form of energies of other $n$-agglomerates that contain, at least, one particle from the agglomerate A and one particle from the agglomerate B. 
Eq. (6) applied to one mole of pure solution component $A_{i}$ reduces to

$$
g_{i}^{0}=\frac{z^{n} N^{0}}{n} g_{n ; k_{i}=n}
$$

Here, $g_{n ; k_{i}=n}=g_{n ;} \underbrace{0, \ldots 0}_{i-1}, n, 0, \ldots, 0$ is the conditional average non-configurational Gibbs free energy of an $n$-agglomerate, provided that the agglomerate consists of particles of type $A_{i}$ only, and $g_{i}^{0}$ is the molar Gibbs free energy of the pure solution component $A_{i}$.

Since the non-configurational molar Gibbs free energy $g^{\text {nc }}$ does not take into account configurational degrees of freedom, the configurational entropy term should be added to give the total molar Gibbs free energy of the solution $g$,

$$
\begin{aligned}
g= & g^{\mathrm{nc}}+R T \sum_{i=1}^{r} x_{i} \ln x_{i}= \\
& \frac{z_{n} N^{0}}{n}\left(\sum_{k_{1}+\ldots+k_{r}=n} \frac{n !}{k_{1} ! \ldots k_{r} !} x_{1}^{k_{1}} \ldots x_{r}^{k_{r}} g_{n ; k_{1}, \ldots, k_{r}}\right)+R T \sum_{i=1}^{r} x_{i} \ln x_{i} .
\end{aligned}
$$

Here, $R T \sum_{i=1}^{r} x_{i} \ln x_{i}$ is the conventional entropy term in the Bragg-Williams approximation.

Consider the following generalised quasichemical reaction

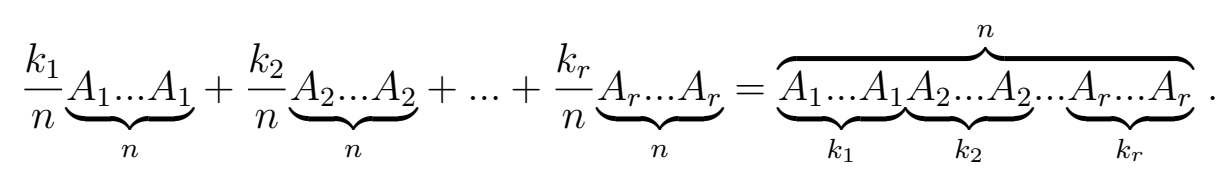

In the reaction Eq. (9) one mole of $n$-agglomerates, each of which consists of $k_{1}$ particles of type $A_{1}, k_{2}$ particles of type $A_{2}$ and so on, such that $k_{1}+k_{2}+\ldots+k_{r}=n$, is forming from $k_{1} / n$ moles of $n$-agglomerates, which consist only of particles of type $A_{1}, k_{2} / n$ moles of $A_{2}$-particles $n$-agglomerates and so on. The order in the sequence $k_{1}, \ldots, k_{r}$ is determined by the numbering of the solution components.

The molar Gibbs free energy $\Delta g_{\text {g.r. }}$ of the generalised quasichemical reaction Eq. (9) 
can be expressed as follows:

$$
\Delta g_{\text {g.r. }}=N^{0} \Delta g_{n ; k_{1}, \ldots, k_{r}}=N^{0}\left(g_{n ; k_{1}, \ldots, k_{r}}-\sum_{i=1}^{r} \frac{k_{i}}{n} g_{n ; k_{i}=n}\right)
$$

where $\Delta g_{n ; k_{1}, \ldots, k_{r}}$ is the Gibbs free energy change per one act of the reaction. If for a given $i, k_{i}=n$ while $k_{j}=0$ for any $j \neq i$, then there is no reaction in Eq. (9) and $\Delta g_{n ; k_{i}=n} \equiv 0$.

Using Eq. (10), the sum in brackets in Eq. (8) can be rearranged as follows:

$$
\begin{aligned}
& \sum_{k_{1}+\ldots+k_{r}=n} \frac{n !}{k_{1} ! \ldots k_{r} !} x_{1}^{k_{1}} \ldots x_{r}^{k_{r}} g_{n ; k_{1}, \ldots, k_{r}}= \\
& \sum_{k_{1}+\ldots+k_{r}=n} \frac{n !}{k_{1} ! \ldots k_{r} !} x_{1}^{k_{1}} \ldots x_{r}^{k_{r}} \Delta g_{n ; k_{1}, \ldots, k_{r}}+\sum_{k_{1}+\ldots+k_{r}=n}\left(\frac{n !}{k_{1} ! \ldots k_{r} !} x_{1}^{k_{1}} \ldots x_{r}^{k_{r}} \sum_{i=1}^{r} \frac{k_{i}}{n} g_{n ; k_{i}=n}\right) .
\end{aligned}
$$

The second term on the right in Eq. (11) can be rearranged as follows:

$$
\begin{aligned}
& \sum_{k_{1}+\ldots+k_{r}=n}\left(\frac{n !}{k_{1} ! \ldots k_{r} !} x_{1}^{k_{1}} \ldots x_{r}^{k_{r}} \sum_{i=1}^{r} \frac{k_{i}}{n} g_{n ; k_{i}=n}\right)= \\
& \sum_{j=0}^{n}\left(\sum_{\begin{array}{c}
n+\ldots+k_{i-1}+ \\
k_{i+1}+\ldots+k_{r}=n-j
\end{array}} \frac{n !}{k_{1} ! \ldots k_{i-1} ! j ! k_{i+1} ! \ldots k_{r} !} x_{1}^{k_{1}} \ldots x_{i-1}^{k_{i-1}} x_{i}^{j} x_{i+1}^{k_{i+1}} \ldots x_{r}^{k_{r}} \sum_{i=1}^{r} \frac{j}{n} g_{n ; k_{i}=n}\right)= \\
& \sum_{j=0}^{n} \sum_{i=1}^{r} \frac{j}{n} g_{n ; k_{i}=n} \frac{n ! x_{i}^{j}}{j !(n-j) !}\left(\sum_{\begin{array}{c}
k_{1}+\ldots+k_{i-1}+ \\
k_{i+1}+\ldots+k_{r}=n-j
\end{array}} \frac{(n-j) !}{k_{1} ! \ldots k_{i-1} ! k_{i+1} ! \ldots k_{r} !} x_{1}^{k_{1}} \ldots x_{i-1}^{k_{i-1}} x_{i+1}^{k_{i+1}} \ldots x_{r}^{k_{r}}\right)= \\
& \sum_{i=1}^{r} \sum_{j=0}^{n} \frac{j}{n} g_{n ; k_{i}=n} \frac{n ! x_{i}^{j}\left(1-x_{i}\right)^{n-j}}{j !(n-j) !}=\sum_{i=1}^{r} x_{i} g_{n ; k_{i}=n} \sum_{p=0}^{n-1} \frac{(n-1) ! x_{i}^{p}\left(1-x_{i}\right)^{n-1-p}}{p !(n-1-p) !}=\sum_{i=1}^{r} x_{i} g_{n ; k_{i}=n} .
\end{aligned}
$$

Substitution of Eqs. (11) and (12) to Eq. (8) gives

$$
\begin{aligned}
g & =\frac{z_{n} N^{0}}{n}\left(\sum_{i=1}^{r} x_{i} g_{n ; k_{i}=n}\right)+R T \sum_{i=1}^{r} x_{i} \ln x_{i} \\
& +\frac{z_{n} N^{0}}{n}\left(\sum_{k_{1}+\ldots+k_{r}=n} \frac{n !}{k_{1} ! \ldots k_{r} !} x_{1}^{k_{1}} \ldots x_{r}^{k_{r}} \Delta g_{n ; k_{1}, \ldots, k_{r}}\right) .
\end{aligned}
$$

Substitution of Eq. (7) to Eq. (13) gives

$$
g=\sum_{i=1}^{r} x_{i} g_{i}^{0}+R T \sum_{i=1}^{r} x_{i} \ln x_{i}+\sum_{k_{1}+\ldots+k_{r}=n}\left(\frac{n !}{k_{1} ! \ldots k_{r} !} \frac{z_{n} N^{0}}{n} \Delta g_{n ; k_{1}, \ldots, k_{r}}\right) x_{1}^{k_{1}} \ldots x_{r}^{k_{r}}
$$


Using the notation

$$
c_{n ; k_{1}, \ldots, k_{r}}=\frac{n !}{k_{1} ! \ldots k_{r} !} \frac{z_{n} N^{0}}{n} \Delta g_{n ; k_{1}, \ldots, k_{r}}
$$

Eq. (14) can be reduced to the power series multicomponent Polynomial solution model

$$
g=\sum_{i=1}^{r} x_{i} g_{i}^{0}+R T \sum_{i=1}^{r} x_{i} \ln x_{i}+\sum_{k_{1}+\ldots+k_{r}=n} c_{n ; k_{1}, \ldots, k_{r}} x_{1}^{k_{1}} \ldots x_{r}^{k_{r}}
$$

As demonstrated above, terms of type $c_{n ; k_{1}, \ldots, k_{r}} x_{1}^{k_{1}} \ldots x_{r}^{k_{r}}$ with $k_{1}+\cdots+k_{r}=n$ in the expression for the molar excess Gibbs free energy correspond to $n$-body interactions between mixing particles. If a polynomial of the degree $n$ is used to express the molar excess Gibbs free energy of a solution phase, it indicates that, as a minimum, $n$-body interactions are involved. From this standpoint, the degree of the polynomial used to describe a higher order system should be, at least, equal to the maximal degree of the polynomials used for the description of bounding lower order systems. Note also that all possible combinations of the solution components in the higher order system should be considered. For example, if a forth degree polynomial is used to describe one or more of the bounding ternary systems, the term $c_{4 ; 1,1,1,1} x_{1} x_{2} x_{3} x_{4}$ is required in the quaternary system.

The above treatment is valid for an arbitrary number of solution components and for an arbitrary number of interacting particles. The analysis carried out follows the BraggWilliams based line of reasoning similar to that in the Regular solution model for binary systems [9]. Eq. (15) establishes a physically meaningful link between the coefficients of the power series multicomponent solution model represented by Eq. (16) on the one hand and the Gibbs free energies of the generalised quasichemical reactions Eq. (9) on the other. This relationship provides a theoretical background for the ternary and multicomponent interaction parameters.

Note that the issue of multi-body interactions is addressed in the Cluster Variation 
Method $[18,19]$ and in the Central Atom model $[20,21]$. These models take into account spatial arrangement of particles and go beyond the assumptions of random mixing (BraggWilliams approximation). Another distinction of the approach suggested in the present study is that neither the Cluster Variation Method nor the Central Atom model consider the generalised quasichemical reactions.

\section{Estimation of the Ternary Interaction Parameter}

As pointed out by Helffrich and Wood [7] and by Ganguly [12], it is impossible, in general, to determine the ternary interaction parameters from the binary parameters only. However, the probabilistic interpretation of the parameters presented in the previous section provides a method for estimating the ternary interaction parameter. This estimation allows incorporating the power series model into the Calphad approach. In simple cases considered below, the value of the ternary parameter can be predicted using physically reasonable assumptions.

\subsection{The Example Presented by Chartrand and Pelton}

In Section 3.2, the example of a hypothetical ternary system $A_{1}-A_{2}-A_{3}$ presented by Chartrand and Pelton [3] was briefly discussed. Let us now consider the example in more detail using the treatment of the generalised quasichemical reactions Eq. (9). Since the binaries $A_{1}-A_{2}$ and $A_{1}-A_{3}$ behave subregularly, and the binary $A_{2}-A_{3}$ is ideal, it could be assumed that three-body interactions are sufficient to describe the Gibbs free energy both in the binaries and in the ternary system.

In the considered example the molar excess Gibbs free energy of the binary solution $A_{1}-A_{2}$ is given by ${ }^{E} g_{12}\left(x_{1}, x_{2}\right)=-50\left(1-x_{1}\right) x_{1} x_{2}=-50 x_{1} x_{2}^{2} \mathrm{~kJ} / \mathrm{mol}$. This term is related to the reaction

$$
\frac{1}{3} A_{1} A_{1} A_{1}+\frac{2}{3} A_{2} A_{2} A_{2}=A_{1} A_{2} A_{2}
$$


According to Eq. (15), the Gibbs free energy change per one act of the reaction Eq. (17) $\Delta g_{3 ; 1,2,0}$ is given by $\Delta g_{3 ; 1,2,0}=-50 /\left(z_{3} N^{0}\right) \mathrm{kJ}$. A similar consideration applied to the binary $A_{1}-A_{3}$ gives $\Delta g_{3 ; 1,0,2}=-50 /\left(z_{3} N^{0}\right) \mathrm{kJ}$, where $\Delta g_{3 ; 1,0,2}$ is the Gibbs free energy change per one act of the following reaction

$$
\frac{1}{3} A_{1} A_{1} A_{1}+\frac{2}{3} A_{3} A_{3} A_{3}=A_{1} A_{3} A_{3}
$$

The Gibbs free energies of the reactions

$$
\begin{aligned}
& \frac{2}{3} A_{1} A_{1} A_{1}+\frac{1}{3} A_{2} A_{2} A_{2}=A_{1} A_{1} A_{2} \text { and } \\
& \frac{2}{3} A_{1} A_{1} A_{1}+\frac{1}{3} A_{3} A_{3} A_{3}=A_{1} A_{1} A_{3}
\end{aligned}
$$

are assumed to be zero in the present example.

As soon as three-body interactions are assumed, the ternary interaction term of type $c_{3 ; 1,1,1} x_{1} x_{2} x_{3}$ should also be considered for the ternary system. This term is related to the reaction

$$
\frac{1}{3} A_{1} A_{1} A_{1}+\frac{1}{3} A_{2} A_{2} A_{2}+\frac{1}{3} A_{3} A_{3} A_{3}=A_{1} A_{2} A_{3}
$$

The Gibbs free energy of the reaction Eq. (21) is not known. However, under reasonable assumptions, it can be obtained from the analysis of exchange reactions.

The ideal behaviour of the binary $A_{2}-A_{3}$ implies that the Gibbs free energies of the exchange reactions

$$
\begin{aligned}
& \frac{2}{3} A_{2} A_{2} A_{2}+\frac{1}{3} A_{3} A_{3} A_{3}=A_{2} A_{2} A_{3} \text { and } \\
& \frac{1}{3} A_{2} A_{2} A_{2}+\frac{2}{3} A_{3} A_{3} A_{3}=A_{2} A_{3} A_{3}
\end{aligned}
$$


are zero. It follows that the Gibbs free energies of the reactions

$$
\begin{aligned}
& A_{2}\left(A_{2} A_{2}\right)+A_{2}\left(A_{3} A_{3}\right)=A_{2}\left(A_{2} A_{3}\right)+A_{2}\left(A_{2} A_{3}\right) \text { and } \\
& A_{3}\left(A_{2} A_{2}\right)+A_{3}\left(A_{3} A_{3}\right)=A_{3}\left(A_{2} A_{3}\right)+A_{3}\left(A_{2} A_{3}\right) .
\end{aligned}
$$

are also zero. This implies that neither $A_{2}$ nor $A_{3}$ particles affect the Gibbs free energy change in the pair-exchange reaction

$$
\left(A_{2} A_{2}\right)+\left(A_{3} A_{3}\right)=2\left(A_{2} A_{3}\right)
$$

Hence, it is reasonable to assume that $A_{1}$ particles also have no effect on the Gibbs free energy of the reaction Eq. (26). In this case, the Gibbs free energy of the following exchange reaction

$$
A_{1}\left(A_{2} A_{2}\right)+A_{1}\left(A_{3} A_{3}\right)=A_{1}\left(A_{2} A_{3}\right)+A_{1}\left(A_{2} A_{3}\right)
$$

is zero too. This implies that the Gibbs free energy change $\Delta g_{3 ; 1,1,1}$ per one act of the reaction Eq. (21) is equal that of the reaction Eq. (17) or Eq. (18):

$$
\Delta g_{3 ; 1,1,1}=\Delta g_{3 ; 1,0,2}=\Delta g_{3 ; 1,2,0}=-\frac{50}{z_{3} N^{0}} \mathrm{~kJ} \equiv \Delta g
$$

Therefore, using Eq. (15) and Eq. (28) the molar excess Gibbs free energy in the ternary system $A_{1}-A_{2}-A_{3}$ is expressed as

$$
\begin{aligned}
{ }^{E} g_{123}\left(x_{1}, x_{2}, x_{3}\right)= & \frac{z_{3} N^{0}}{3}\left(3 x_{1} x_{2}^{2} \Delta g_{3 ; 1,2,0}+3 x_{1} x_{3}^{2} \Delta g_{3 ; 1,0,2}+6 x_{1} x_{2} x_{3} \Delta g_{3 ; 1,1,1}\right)= \\
& z_{3} N^{0} \Delta g x_{1}\left(x_{2}^{2}+x_{3}^{2}+2 x_{2} x_{3}\right)=-50 x_{1}\left(1-x_{1}\right)^{2} \mathrm{~kJ} .
\end{aligned}
$$

According to Eq. (29), the ternary molar excess Gibbs free energy only depends on $x_{1}$, as it has been expected initially.

Note also that under the assumptions made, $z_{n}$ is the same for all systems. As demon- 
strated above, it is obtained from known coefficients of the polynomial expansion of the molar excess Gibbs free energy of lower order systems and used for the coefficients in the higher order system.

A ternary system with two identical subregular binaries and with ideal third binary has already been analysed by Hillert [5] and later by Cheng and Ganguly [2]. In both cases, the result similar to Eq. (29) has been initially assumed, and the ternary interaction parameter was selected to obtain the desired result. In the present study, this result is derived from the reasonable assumption using the proposed probabilistic interpretation and the analysis of the generalised quasichemical reactions.

\subsection{A Case of the Regular Binary $A_{2}-A_{3}$}

A ternary system with the subregular binaries has been analysed by Cheng and Ganguly [2], where the molar excess Gibbs free energy in the ternary system has been expressed as

$$
{ }^{E} g_{123}\left(x_{1}, x_{2}, x_{3}\right)=\sum_{i \neq j} x_{i} x_{j}\left(\hat{w}_{i j} x_{j}+\hat{w}_{j i} x_{i}\right)+x_{1} x_{2} x_{3}\left(\frac{1}{2} \sum_{i \neq j}\left(\hat{w}_{i j}+\hat{w}_{j i}\right)+c_{123}\right) .
$$

Here, $\hat{w}_{i j}$ and $\hat{w}_{j i}$ are constants used to describe the behaviour of the bounding binaries. The following estimate of the parameter $c_{123}$ has been proposed

$$
c_{123} \approx \sum_{i \neq j \neq k} \sigma_{i j} \sigma_{i k}\left[\left(\hat{w}_{i j}-\hat{w}_{j i}\right) \frac{x_{j}}{x_{j}+x_{k}}+\left(\hat{w}_{i k}-\hat{w}_{k i}\right) \frac{x_{k}}{x_{j}+x_{k}}\right]
$$

where $\sigma_{i j}=\sigma_{j i}=0$ for $i=j$, and $\sigma_{i j}=\sigma_{j i}=1$ otherwise.

However, as demonstrated above, the ternary interaction parameter is related to the Gibbs free energy of the correspondent generalised quasichemical reaction Eq. (9) which does not depend on the composition. Moreover, the terms of type $x_{j} /\left(x_{j}+x_{k}\right)$ used in Eq. (31) seem to be problematic. Indeed, as demonstrated by Brynestad [13] such terms could lead to singularities in the partial derivatives of the Gibbs free energy.

The approach used in the previous section for the ideal binary $A_{2}-A_{3}$ can be extended to the case of regular binary. It can be demonstrated that the regular behaviour of the binary 
$A_{2}-A_{3}$ implies that the Gibbs free energies of the exchange reactions Eq. (24) and Eq. (25) are equal to $\Delta g_{3 ; 0,1,2}=\Delta g_{3 ; 0,2,1}$. With the same reasoning as was used in the previous section, it could be assumed that the Gibbs free energy of the exchange reaction Eq. (27) is also $\Delta g_{3 ; 0,1,2}$. It can be derived that $\Delta g_{3 ; 1,1,1}=1 / 2\left(\Delta g_{3 ; 0,1,2}+\Delta g_{3 ; 1,2,0}+\Delta g_{3 ; 1,0,2}\right)$. The ternary interaction parameter is then obtained from Eq. (15).

It should be noted that the assumed similarity of the exchange reactions is not necessarily valid for all ternary systems. In some systems the Gibbs free energy of the pair exchange reaction Eq. (26) could be affected by $A_{1}$ particles. However, the value of the ternary interaction parameter obtained under the above mentioned assumption can still be used as a preliminary approximation. This value can then be refined using the available ternary data. The examples considered above support the viability of the approach suggested in the present study. Moreover, the proposed methodology seems to be amenable to further extensions towards estimating multicomponent interaction parameters from the bounding binaries.

\section{Impact of the Ternary Term}

Recently, Janz and Schmid-Fetzer [22] investigated the impact of the ternary interaction parameter on activities of solution components and found this impact to be counterintuitive compared to that of the conventional regular parameter. It has been recommended by Janz and Schmid-Fetzer [22] to use the ternary term with precautions or not to use it at all, but rather to improve on the extrapolation model or on the description of bounding binary systems.

Note that the observed effect is related rather to a type of term than to a value of the parameter. The ternary term is proportional to the probability $p_{123}$ to pick up a triplet $A_{1} A_{2} A_{3}$ at random, while the conventional regular term is proportional to the probability $p_{12}$ of choosing a pair $A_{1} A_{2}$. These probabilities have the multinomial distribution and, as functions of composition, behave differently for different terms. From this point of 
view, the dissimilar impacts of different terms on the activities of solution components are rather expected than unusual.

To clarify the difference, consider a hypothetical $r$-component solution $A_{1}-\ldots-A_{q}-$ $\ldots-A_{r}$. Assume that for arbitrary $q$, such that $2 \leq q \leq r$, the $q$-component and $q$-body term $L_{q} x_{1} \ldots x_{q}$ is the only molar excess Gibbs free energy term. The parameter $L_{q}$ is determined by Eq. (15). Let $\gamma_{1}$ be the activity coefficient of the component $A_{1}$. One can verify that

$$
R T \ln \gamma_{1}=L x_{2} \ldots x_{q}\left(x_{1}(2-q)+x_{2}+\ldots+x_{q}\right)
$$

Eq. (32) demonstrates that $\ln \gamma_{1}$ does not change its sign and independent of $x_{1}$ for conventional regular term $(q=2)$. However, for more complex terms $(q>2)$, which account for the Gibbs free energies of multi-body interactions, $\ln \gamma_{1}$ changes its sign at $x_{1}=\left(x_{2}+\ldots+x_{q}\right) /(q-2)$.

The present author is of opinion that the impact of the ternary term on the activities described by Janz and Schmid-Fetzer [22] is not extraordinary. The ternary term should always be used in a ternary system, when three-body interactions are involved.

\section{Conclusions}

A probabilistic interpretation of the parameters of the power series Polynomial solution model is proposed in the present study. The presented treatment explicitly relates the ternary and multicomponent interaction parameters with the Gibbs free energies of the generalised quasichemical reactions and provides a theoretical justification for such parameters. The methodology of estimating the ternary interaction parameter from the binary ones is also presented. The methodology provides a way, in which the power series multicomponent model, where no projection is required, could be incorporated into the Calphad approach. 


\section{Acknowledgment}

The author is grateful to Dr. Igor Vladimirov for helpful discussions on the subject.

\section{References}

[1] M. Margules, Uber die zusammensetzung der gesiittigten dampfe von misehungen, Sitzb. d. mathem. naturw. Cl. 104 (1895) 1243-1278.

[2] W. Cheng, J. Ganguly, Some aspects of multicomponent excess free energy models with subregular binaries, Geochim. Cosmochim. Acta 58 (1994) 3763-3767.

[3] P. Chartrand, A. D. Pelton, On the choice of "geometric" thermodynamic models, J. Phase Equilib. 21 (2) (2000) 141-147.

[4] A. D. Pelton, A general "geometric" thermodynamic model for multicomponent solutions, Calphad 25 (2) (2001) 319-328.

[5] M. Hillert, Empirical methods of predicting and representing thermodynamic properties of ternary solution phases, Calphad 4 (1980) 1-12.

[6] K. Wohl, Thermodynamic evaluation of binary and ternary liquid systems, T. Am. Inst. Chem. Eng. 42 (1946) 215-249.

[7] G. Helffrich, B. Wood, Subregular model for multicomponent solutions, Am. Mineral. 74 (1989) 1016-1022.

[8] B. Mukhopadhyay, S. Basu, M. Holdaway, A dscussion of Margules-type formulations for multicomponent solutions with a generalized approach, Geochim. Cosmochim. Acta 57 (1993) 277-283.

[9] M. Hillert, Phase Equilibria, Phase Diagrams and Phase Transformations: Their Thermodynamic Basis, Cambridge University Press, 1998. 
[10] R. Ganesan, S. V. Varamban, A parabolic model to estimate ternary thermodynamic properties, Calphad 21 (1997) 509-519.

[11] A. D. Pelton, Thermodynamic and phase diagrams of materials, in: G. Kostorz (Ed.), Phase Transformations in Materials, Wiley-VCH, 2001.

[12] J. Ganguly, Thermodynamic modelling of solid solutions, in: C. Geiger (Ed.), Solid Solutions in Silicate and Oxide Systems of Geological Importance, Vol. 3 of Notes in Mineralogy, European Mineralogical Union, 2001, pp. 37-69.

[13] J. Brynestad, Incompatibility between the Kohler method and the subregular model, Calphad 5 (1981) 103-104.

[14] L. Kaufman, Comment on "Incompatibility between the Kohler method and the subregular model, Calphad 5103 (1981)", Calphad 5 (1981) 225-226.

[15] M. Hillert, B. Sundman, Comment on "Incompatibility between the Kohler method and the subregular model", Calphad 6 (1982) 65.

[16] R. A. Howald, B. N. Row, Muggianu and Toop-Muggianu interpolations comment of a comment by L. Kaufman (Calphad 5, 225 (1981)) on Brynestad's paper (Calphad, 5, 103, (1981)), Calphad 6 (1982) 57-63.

[17] W. Feller, An Introduction to Probability Theory and Its Applications, Vol. 1, John Wiley \& Sons, 1971.

[18] R. Kikuchi, A theory of cooperative phenomena, Phys. Review 81 (1951) 988-1003.

[19] A. Pelizzola, Cluster variation method in statistical physics and probabilistic graphical models, J. Phys. A 38 (2005) R309-R339.

[20] C. H. P. Lupis, J. F. Elliott, Prediction of enthalpy and entropy interaction coefficients by the "Central Atoms" theory, Acta Metall. 15 (1967) 265-267. 
[21] E.-H. Foo, C. H. P. Lupis, The "Central Atoms" model of multicomponent interstitial solutions and its applications to carbon and nitrogen in iron alloys, Acta Metall. 21 (1973) 1409-1430.

[22] A. Janz, R. Schmid-Fetzer, Impact of ternary parameters, Calphad 29 (2005) 37-39. 